\title{
Composición y distribución del macrobentos en la Ensenada de Sechura, Piura, Perú
}

\section{Composition and distribution of macrobenthos in the Bay of Sechura, Piura, Peru}

\author{
Williams Yupanqui', Luis Quipúzcoa', Robert Marquina', Federico Velazco², Ed- \\ gardo Enríquez', Dimitri Gutiérrez
}

\begin{abstract}
1 Laboratorio de Bentos Marino. Dirección de Investigaciones Oceanográficas. Instituto del Mar del Perú.

${ }^{2}$ Laboratorio de Geología Marina. Dirección de Investigaciones Oceanográficas. Instituto del Mar del Perú.

Email Williams Yupanqui:williamsfyc@yahoo. com
\end{abstract}

Presentado: $\quad$ 02/05/2006 Aceptado: $\quad 01 / 03 / 2006$

\section{Resumen}

En el presente trabajo se describe la distribución espacial de la macrofauna bentónica en la Ensenada de Sechura, Piura $\left(5^{\circ} 12^{\prime}-5^{\circ} 50^{\prime} \mathrm{S}\right.$ y $\left.81^{\circ} 12^{\prime}-80^{\circ} 51^{\prime} \mathrm{W}\right)$. Fueron consideradas 23 estaciones distribuidas entre los 5 y 72 m, muestreadas al finalizar el evento El Niño 1997-98 (julio 1998). La temperatura, el oxigeno disuelto y el porcentaje de arena mostraron una tendencia decreciente desde las estaciones someras hasta las más profundas. Contrariamente la materia orgánica, el número de especies y la equidad (J') aumentaron con la profundidad. El grupo taxonómico que alcanzó la mayor densidad fue Polychaeta (81\%). En las estaciones someras (5 -- $11 \mathrm{~m}$ ) la composición especifica fue muy variada, tendiendo a dominar Polydora socialis (54\%) en sustratos arenosos y rocosos. En las estaciones entre los 21 y $35 \mathrm{~m}$, dominaron los poliquetos de la familia Capitellidae (17\%), mientras que en las más profundas $(42-72 \mathrm{~m}$ ) dominó el poliqueto Paraprionospio pinnata (27\%). Asimismo, los gremios tróficos de poliquetos mejor representados fueron los depositívoros superficiales y los consumidores de interfase. Al comparar los resultados con información obtenida en junio de 1997 se encontraron diferencias en las especies dominantes, particularmente en el estrato intermedio de profundidad, en tanto no se detectaron diferencias significativas en los parámetros comunitarios.

Palabras Clave: Ensenada de Sechura, Macrofauna, Polychaeta, Batimetría, Sedimento.

\section{Abstract}

It was analyzed the spatial distribution of the benthic macrofauna of the Bay of Sechura $\left(5^{\circ} 12\right.$ - $5^{\circ} 50^{\prime} \mathrm{S}$ and $81^{\circ} 12^{\prime}-80^{\circ} 51^{\prime} \mathrm{W}$ ) right after the end the 1997-98 El Niño. Temperature, dissolved oxygen, sand content (\%), exhibited a decreasing trend from shallow to deep stations, while organic matter content, equitability and diversity increased with depth. The Polychaeta were the most abundant taxonomic group (81\%). The composition was very heterogenous at shallower depths $(5-11 \mathrm{~m})$, and the polychaete Polydora socialis tended to dominate in sandy and rocky bottoms with $54 \%$. Polychaetes from the family Capitellidae (17\%) dominated at intermediate depths $(21$ - $35 \mathrm{~m}$ ), while the spionid polychaete Paraprionospio pinnata $(27 \%)$ was the dominant species at deeper locations $(42-72 \mathrm{~m})$. The dominant feeding guilds were surface deposit feeders and interface feeders. In comparison with information collected at the onset of El Niño 1997-98, there were differences in the composition of dominant species, especially at intermediate depths, but there were not found significant differences in the community parameters.

Key words: Bay of Sechura, Macrofauna, Polychaeta, Bathymetric distribution, Sediment

\section{Introducción}

La ensenada de Sechura (comprendida entre Punta Gobernador y Punta Aguja) se ubica en la zona de convergencia de aguas frías de la corriente costera peruana y de aguas cálidas ecuatoriales superficiales. Es una importante área costera con altos contenidos de nutrientes y elevada producción de fitoplancton, zooplancton e ictioplancton (Jacinto et al., 1997; Ayon y Aronés, 1997). Estos factores, entre otros, permiten el desarrollo de diversos recursos hidrobiológicos que son aprovechados comercialmente, como la concha de abanico y otros que sustentan la pesquería artesanal del lugar (Flores et al., 1994; 1997; 1998). Producto de la dinámica de la zona costera, de la relativa poca profundidad y de la alta producción biológica, los sedimentos están distribuidos heterogéneamente y presentan diversos tipos, como arenoso, limo-arenoso y fango (Delgado y Gomero, 1988).

La información sobre la macrofauna que habita la Ensenada de Sechura es aún escasa, resultado en su mayoría de muestreos de oportunidad. Salzwedel et al. (1988) presentaron datos de parámetros comunitarios de la macrofauna entre los 5 y $6^{\circ} \mathrm{S}$, pero sin precisar la ubicación de las estaciones. Por otra parte, Mayor (1996), reportó valores de densidad y de biomasa entre los 50 y $80 \mathrm{~m}$ de profundidad. Asimismo, cierta información adicional de algunas estaciones someras o profundas provienen de los cruceros de evaluación de recursos demersales (Mayor et al., 1997; L. Quipúzcoa com. pers.) o asociados a estudios de poblaciones de concha de abanico (Rubio et al., 1996; Rubio y Taipe, 1996; Rubio et al., 1997; Tafur et al., 2000), ambos realizados por el Instituto del Mar del Perú (IMARPE).

La importancia de las comunidades bentónicas radica en el rol que cumplen dentro de los ciclos biogeoquímicos y en la cadena trófica del ecosistema marino y de ahí la necesidad de conocer el comportamiento de tales comunidades frente a los cambios oceanográficos estacionales, anuales o interanuales. Entre 1997 y 1998 se produjeron cambios importantes en la estructura comunitaria de la macrofauna a lo largo del litoral peruano, ocasionados por el aumento de la temperatura y el oxígeno en el fondo asociado al evento extraordinario El Niño 1997-1998 (EN 1997-98). Este evento perturbó en diverso grado las comunidades macrofaunales de las bahías de Paita 
(5 S) y San José (7o S) (Gutiérrez et al., 2005), Ancón (11º S) (Tarazona et al., 2000), entre otras.

Los objetivos del presente trabajo son: caracterizar la comunidad macrozoobentónica de fondo blando de la Ensenada de Sechura y determinar los factores fisico-químicos del agua y sedimento que condicionan su estructura y distribución, en el período inmediatamente posterior a la finalización de EN 1997-98. Se hipotetiza que factores asociados a la profundidad y las características del sedimento controlan la distribución y estructura de la macrofauna en la Ensenada de Sechura.

\section{Material y métodos}

La Ensenada de Sechura, en Piura, se encuentra ubicada en la costa norte del Perú, entre los $5^{\circ} 12^{\prime}$ y $5^{\circ} 50^{\prime} S$ y $81^{\circ} 12^{\prime}$ y $80^{\circ} 51^{\prime} \mathrm{W}$. La zona litoral está bordeada por playas arenosas y en los extremos por acantilados. En la zona sublitoral las mayores profundidades se encuentran en la parte central $(-80 \mathrm{~m})$. Hacia el sur la ensenada está influenciada por la llegada de agua dulce proveniente del Estuario de Virrilá y hacia el este por la bocana de San Pedro, donde desemboca el río Piura.

El muestreo se realizó entre el 7 y 12 de julio de 1998, en 23 estaciones aplicando un diseño por transectas perpendiculares a la costa, a profundidades entre 5 y $72 \mathrm{~m}$ (Fig. 1).

Las muestras de agua en el fondo fueron colectadas con una botella Niskin de 5 L de capacidad. La concentración de oxígeno disuelto se determinó mediante el método de Winkler modificado por Carriet and Carpenter (1966) y la salinidad de fondo usando un salinómetro Kahlsico R-10. La temperatura se registró en el lugar mediante un termómetro de inversión.

Las muestras de sedimento fueron tomadas por triplicado con una draga tipo van Veen de $0,05 \mathrm{~m}^{2}$ de superficie de colecta.

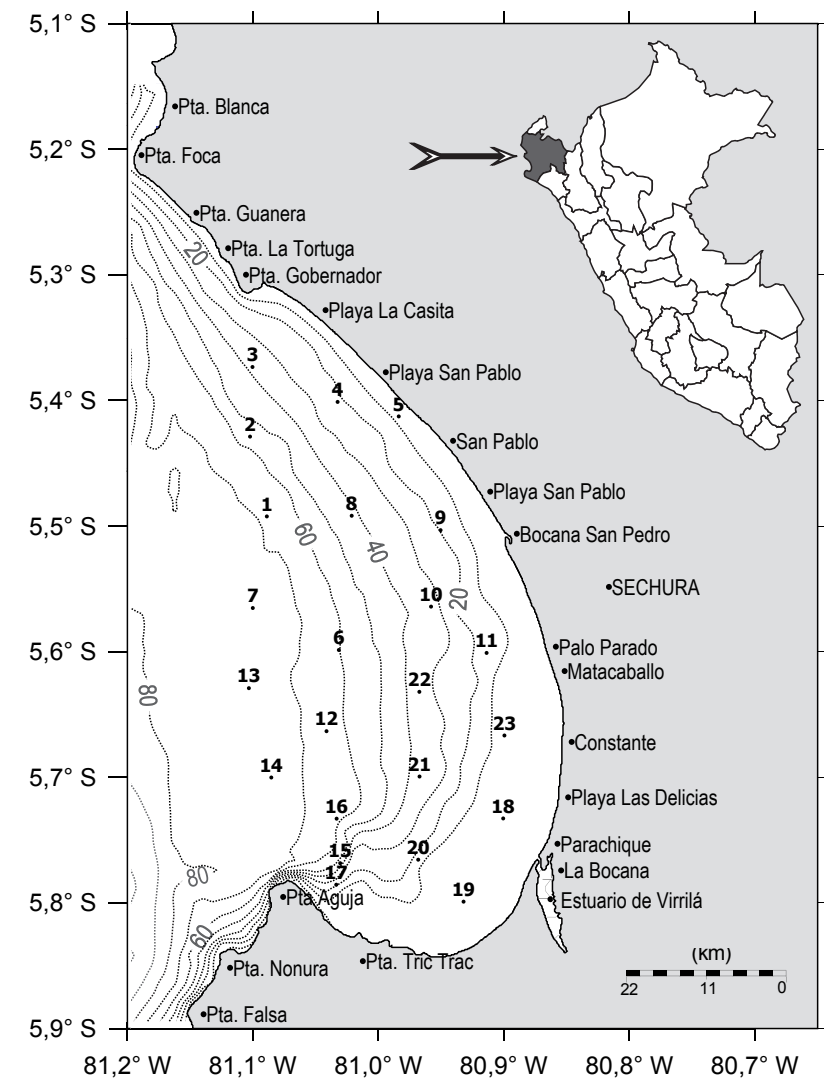

Figura 1. Carta de posiciones de las estaciones en la Ensenada de Sechura, julio de 1998.
Además se separó una submuestra de los 5 primeros centímetros superficiales para los análisis de composición granulométrica (Ingram, 1971; Galehouse, 1971), materia orgánica total y carbonatos totales (Dean, 1974); el resto fue tamizado a través de una malla de $500 \mu \mathrm{m}$ y las muestras fueron preservadas en frascos con formol al 10\% neutralizado con boráx.

El análisis biológico consistió en determinar la composición de la comunidad macrozoobentónica hasta el menor nivel taxonómico posible, determinándose la densidad (número de individuos. 0,05 $\mathrm{m}^{-2}$ ) y la biomasa (peso húmedo en g. 0,05 $\mathrm{m}^{-2}$ ) de los organismos. Los taxa de poliquetos fueron asignados a gremios tróficos en base a literatura (Fauchald \& Jumars, 1979; Gastón, 1987; Maurer et al., 1994; Levin et al., 1997; Gutiérrez, 2000). Las categorías de gremios tróficos utilizadas fueron: a)consumidores de interfase (IF) que pueden mudar su alimentación de filtración a resuspensión, compuestos por la familia Spionidae y Oweniidae; b) depositívoros superficiales (SDF), compuestos por las familias Capitellidae, Paraonidae, Ampharetidae entre otros; c)depositívoros subsuperficiales (SSDF), que incluyen a los Maldanidae, Orbiniidae, Cossuridae entre otros; d) omnívoros depredadores (OP), que albergan a las familias Nereidae, Nephtyidae, Hesionidae, Glyceridae, Syllidae entre otros; y e) suspensívoros (SF), compuestos por la familia Sabellidae, Serpulidae, Sabellariidae, entre otros.

El mapa de distribución espacial fue elaborado con el programa Golden Surfer, versión 8.0. Los datos obtenidos fueron tabulados en matrices tanto para los factores abióticos como para los parámetros bióticos. Estas matrices fueron sometidas a pruebas univariadas y multivariadas.

Se empleó el análisis de varianza no paramétrica de una vía de Kruskal-Wallis (Siegel, 1994) para evaluar las diferencias en los principales parámetros comunitarios (densidad, biomasa, número de especies, diversidad y equidad) entre más de dos grupos, tales como los estratos de profundidad (I, II, III). Para las comparaciones entre pares, se aplicó la prueba no paramétrica de U-MannWhitney (Siegel, 1994), que luego sirvió para generar grupos estadísticamente homogéneos, al nivel de confianza $\mathrm{p}=0,05$.

Para los aspectos cuantitativos de la estructura comunitaria se consideran el número de especies, densidad y biomasa media por estación de muestreo y la desviación estándar. La abundancia por replica por especie por estación de muestreo se empleó para calcular la diversidad empleando el índice de Shannon y Wiener $\left(\mathrm{H}^{\prime}\right)$ y el índice de equidad $\left(\mathrm{J}^{\prime}\right)$ de Pielou, calculados usando $\log _{2}$.

Para determinar el grado de similitudes entre estaciones se realizo un análisis multivariado de clasificación numérica usando el método de clasificación jerárquica aglomerativa en la media ponderada (grupo par no ponderado con promedio aritmético UPGMA) (Sneath y Sokal, 1973) con el índice de Bray-Curtis, para realizar el análisis de ordenación no métrico multidimensional (NMDS) (Prentice, 1977), análisis de ordenamiento que construye un "mapa" multivariable de la ubicación de las muestras.

Para estudiar las relaciones de los parámetros biológicos y los factores abióticos se aplicó el Análisis de Correspondencia Canónica (ACC), de matrices dobles de datos, combinando las 16 especies de mayor dominancia y frecuencia (transformados a raíz cuarta) con la profundidad, temperatura, oxígeno, limo, arcilla, arena y materia orgánica (transformadas a $\log (\mathrm{x}+1)$ ), mediante el programa Pc-Ord v.4.1 (Valentin, 2000). 
El resultado es un diagrama de ordenación donde las especies y sitios son representados por puntos y las variables ambientales por vectores. Cada eje explica una fracción decreciente de la varianza de la correlación de los taxa respecto a las variables ambientales.

Para la comparación de los valores de biomasa con otras bahías, se convirtió el peso húmedo en peso seco libre de cenizas (AFDW) siguiendo la referencia de Brey et al. (1988).

\section{Resultados}

\section{Parámetros físico-químicos del agua de mar}

Los resultados se muestran en la Tabla 1 . El rango de la temperatura de fondo del mar varió entre $16,2^{\circ} \mathrm{C}(\mathrm{E}-13$ y E-15) y 17,7 ${ }^{\circ} \mathrm{C}(\mathrm{E}-5)$, con tendencia a disminuir a mayores profundidades y significativa correlación negativa con esta $\left(r_{s}=-0,86, p<0,001\right.$, $\mathrm{N}=23$ ) (Tabla 4). El rango de la salinidad de fondo estuvo entre 35,05 ups (E-20 y E-21) y 35,23 ups (ups= unidades prácticas de salinidad) (E-22). La concentración de oxígeno disuelto en el fondo varió entre 1,05 mL.L-1 (E-21) y 4,00 mL.L-1 (E-19); los mayores valores se encontraron frente al Estuario de Virrilá y los menores en la parte intermedia, con tendencia a disminuir desde las estaciones someras a las profundas y una correlación negativa con la profundidad $\left(\mathrm{r}_{\mathrm{s}}=-0,50, \mathrm{p}=0,015, \mathrm{~N}=23\right)$ (Tabla 4).

\section{Características sedimentológicas}

La distribución textural de los sedimentos superficiales (Tabla 1) en la Ensenada de Sechura muestra dos zonas principales, la zona norte-centro con texturas areno-limosas y la zona sur con texturas limo-arcillosas. En ambos casos, las extensiones de arena son menores (siempre cercanas a la línea de costa) y también se presentan algunos espacios ocupados por fondos rocosos frente a Parachique y a Matacaballo. El tamaño del grano decrece conforme aumenta la distancia a la costa. A profundidades entre 21 y $35 \mathrm{~m}$ los porcentajes de limo y arcilla aumentan, mientras que a mayor profundidad ( 42 y $72 \mathrm{~m}$ ), solamente aumenta el porcentaje de limo.

En muestra fresca los sedimentos con mayores contenidos de material fino (limo y arcilla) presentan una coloración verdosa. Los componentes observados al estereoscopio son principalmente cuarzo, fragmentos líticos, feldespatos, fragmentos calcáreos (más abundantes cerca de la línea costera desde el centro hacia el norte), minerales oscuros ferromagnesianos, entre otros. La fracción orgánica en los sedimentos estuvo conformada por algas, poliquetos y bivalvos, observados durante el muestreo.

El porcentaje de matería orgánica total (MOT) fluctuó entre $1 \%(\mathrm{E}-20)$ y $7 \%(\mathrm{E}-17)$ frente a Bayóvar (Tabla 1). En el área central (que cubre las estaciones 17, 10, 22, 8 y 21), se observó la concentración de valores más altos, para disminuir hacia las estaciones ubicadas a mayor profundidad.

El porcentaje de carbonato total (CBT) estuvo entre $2 \%$ en el extremo sur de la ensenada (E-20) y 66\% en el extremo norte (E-5) (Tabla 1). Los mayores valores (>15\%) se distribuyen desde el litoral frente a Constante hacia el centro y desde el litoral frente a Playa San Pablo hacia el noreste de la ensenada.

Tabla 1. Parámetros físico-químicos en la ensenada de Sechura, julio de 1998. ( $E=$ estación; $P=$ profundidad (m); T=temperatura $\left({ }^{\circ} \mathrm{C}\right) ; \mathrm{S}=$ salinidad (ups); $\mathrm{OD}=$ oxígeno disuelto $\left(\mathrm{ml}^{\mathrm{L}} \mathrm{L}^{-1}\right) ; \mathrm{MOT}=\%$ matería orgánica total; $\mathrm{CBT}=\%$ carbonatos totales)

\begin{tabular}{|c|c|c|c|c|c|c|c|c|c|c|c|c|c|c|}
\hline & \multirow[b]{2}{*}{ E } & \multirow[b]{2}{*}{$\mathrm{P}$} & \multirow[b]{2}{*}{ Latitud } & \multirow[b]{2}{*}{ Longitud } & \multirow[b]{2}{*}{$\mathrm{T}$} & \multirow[b]{2}{*}{ S } & \multirow[b]{2}{*}{ OD } & \multicolumn{6}{|c|}{ Granulometría } & \multirow[b]{2}{*}{ Textura } \\
\hline & & & & & & & & MOT & CBT & Grava & Arena & Limo & Arcilla & \\
\hline \multirow{6}{*}{ 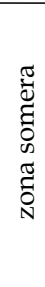 } & E-18 & 5 & $05^{\circ} 43^{\prime} 58,0^{\prime \prime}$ & $80^{\circ} 54^{\prime} 02,0^{\prime \prime}$ & 17,30 & 35,07 & 3,20 & 2,06 & 9,01 & 0,24 & 94,49 & 1,37 & 3,90 & arena \\
\hline & E-19 & 5 & $05^{\circ} 47^{\prime} 56,5^{\prime \prime}$ & $80^{\circ} 55^{\prime} 55,7^{\prime \prime}$ & 17,50 & 35,11 & 4,00 & & & & & & & Rocosa \\
\hline & E-23 & 7 & $05^{\circ} 40^{\prime} 00,3^{\prime \prime}$ & $80^{\circ} 53^{\prime} 58,5^{\prime \prime}$ & 17,30 & 35,06 & 2,30 & 2,33 & 58,29 & 5,17 & 91,10 & 0,36 & 3,37 & arena \\
\hline & E-20 & 10 & $05^{\circ} 45^{\prime} 56,2^{\prime \prime}$ & $80^{\circ} 58^{\prime} 05,8^{\prime \prime}$ & 16,90 & 35,05 & 1,75 & 0,99 & 1,49 & 0 & 92,36 & 1,30 & 6,34 & arena \\
\hline & E-11 & 10 & $05^{\circ} 36^{\prime} 03,7^{\prime \prime}$ & $80^{\circ} 54^{\prime} 49,6^{\prime \prime}$ & 17,40 & 35,10 & 3,50 & & & & & & & Rocosa \\
\hline & E-5 & 11 & $05^{\circ} 24^{\prime} 45,9^{\prime \prime}$ & $80^{\circ} 59^{\prime} 01,6^{\prime \prime}$ & 17,70 & 35,07 & 1,85 & 3,09 & 65,59 & 0,05 & 96,76 & 0,96 & 2,23 & arena \\
\hline \multirow{7}{*}{ 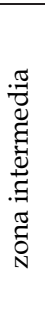 } & E-4 & 21 & $05^{\circ} 24^{\prime} 04,4^{\prime \prime}$ & $81^{\circ} 01^{\prime} 57,0^{\prime \prime}$ & 17,30 & 35,14 & 1,85 & 1,91 & 3,96 & 0,00 & 67,63 & 22,36 & 10,01 & arena limosa \\
\hline & E-17 & 21 & $05^{\circ} 47^{\prime} 07,9^{\prime \prime}$ & $81^{\circ} 02^{\prime} 00,9^{\prime \prime}$ & 16,70 & 35,08 & 2,50 & 6,65 & 3,37 & 0 & 72,92 & 11,55 & 11,53 & arena limosa \\
\hline & E-9 & 25 & $05^{\circ} 30^{\prime} 11,9^{\prime \prime}$ & $80^{\circ} 57^{\prime} 01,6^{\prime \prime}$ & 17,20 & 35,11 & 3,20 & 3,43 & 4,03 & 0 & 25,44 & 61,13 & 13,43 & limo arenoso \\
\hline & E-10 & 28 & $05^{\circ} 33^{\prime} 51,0^{\prime \prime}$ & $80^{\circ} 57^{\prime} 29,0^{\prime \prime}$ & 16,90 & 35,13 & 2,50 & 5,72 & 7,23 & 0,1 & 63,17 & 19,56 & 17,17 & arena limosa \\
\hline & E-22 & 34 & $05^{\circ} 37^{\prime} 55,2^{\prime \prime}$ & $80^{\circ} 58^{\prime} 02,5^{\prime \prime}$ & 17,00 & 35,23 & 1,30 & 4,79 & 15,37 & 0,62 & 83,41 & 5,08 & 10,89 & arena \\
\hline & E-8 & 35 & $05^{\circ} 29^{\prime} 30,6^{\prime \prime}$ & $81^{\circ} 01^{\prime} 16,6^{\prime \prime}$ & 17,00 & 35,11 & 2,90 & 4,73 & 51,21 & 17,11 & 68,98 & 6,04 & 7,87 & arena arcillosa \\
\hline & $\mathrm{E}-21$ & 35 & $05^{\circ} 41^{\prime} 57,9^{\prime \prime}$ & $80^{\circ} 58^{\prime} 02,0^{\prime \prime}$ & 16,90 & 35,05 & 1,05 & 5,25 & 7,83 & 0,03 & 64,50 & 15,76 & 19,71 & arena arcillosa \\
\hline \multirow{10}{*}{ 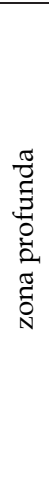 } & E-3 & 42 & $05^{\circ} 22^{\prime} 24,3^{\prime \prime}$ & $81^{\circ} 06^{\prime} 01,2^{\prime \prime}$ & 17,20 & 35,07 & 1,80 & 2,20 & 2,54 & 0,00 & 41,89 & 49,34 & 8,77 & limo arenoso \\
\hline & E-2 & 55 & $05^{\circ} 25^{\prime} 43,8^{\prime \prime}$ & $81^{\circ} 06^{\prime} 08,2^{\prime \prime}$ & 17,00 & 35,15 & 1,85 & 1,74 & 3,25 & 0,00 & 23,47 & 65,41 & 11,12 & limo arenoso \\
\hline & E-6 & 60 & $05^{\circ} 35^{\prime} 55,3^{\prime \prime}$ & $81^{\circ} 01^{\prime} 53,5^{\prime \prime}$ & 16,40 & 35,09 & 1,80 & 2,09 & 2,76 & 0 & 36,87 & 51,23 & 11,90 & limo arenoso \\
\hline & E-16 & 64 & $05^{\circ} 43^{\prime} 58,7^{\prime \prime}$ & $81^{\circ} 01^{\prime} 59,8^{\prime \prime}$ & 16,30 & 35,11 & 2,00 & 1,82 & 2,54 & 0 & 81,57 & 7,34 & 11,09 & arena \\
\hline & E-1 & 65 & $05^{\circ} 29^{\prime} 32,5^{\prime \prime}$ & $81^{\circ} 05^{\prime} 20,0^{\prime \prime}$ & 16,70 & 35,10 & 1,75 & 2,53 & 2,47 & 0,00 & 20,98 & 66,07 & 12,95 & limo arenoso \\
\hline & E-12 & 65 & $05^{\circ} 39^{\prime} 48,0^{\prime \prime}$ & $81^{\circ} 02^{\prime} 28,8^{\prime \prime}$ & 16,40 & 35,11 & 1,75 & 1,76 & 3,76 & 0,00 & 54,81 & 31,90 & 13,29 & arena limosa \\
\hline & E-13 & 70 & $05^{\circ} 37^{\prime} 44,7^{\prime \prime}$ & $81^{\circ} 06^{\prime} 11,5^{\prime \prime}$ & 16,20 & 35,09 & 1,70 & 1,71 & 1,82 & 0,00 & 80,99 & 11,37 & 7,64 & arena \\
\hline & E-14 & 70 & $05^{\circ} 42^{\prime} 00,7^{\prime \prime}$ & $81^{\circ} 06^{\prime} 07,2^{\prime \prime}$ & 16,40 & 35,06 & 1,70 & 2,13 & 2,19 & 0,00 & 76,90 & 12,00 & 11,10 & arena \\
\hline & E-15 & 70 & $05^{\circ} 46^{\prime} 06,3^{\prime \prime}$ & $81^{\circ} 05^{\prime} 49,6^{\prime \prime}$ & 16,20 & 35,08 & 2,50 & 2,23 & 1,98 & 0,00 & 82,74 & 5,33 & 11,93 & arena \\
\hline & E-7 & 72 & $05^{\circ} 33^{\prime} 55,0^{\prime \prime}$ & $81^{\circ} 06^{\prime} 00,3^{\prime \prime}$ & 16,40 & 35,09 & 1,85 & 1,88 & 2,98 & 0 & 61,75 & 26,87 & 11,38 & arena limosa \\
\hline
\end{tabular}


Tabla 2. Densidad y Biomasa por estación y porcentaje e indices de diversidad de la macrofauna en la ensenada de Sechura, julio de 1998. $\mathrm{P}=$ profundidad, $\mathrm{R}=\mathrm{n}^{0}$ replica; $\mathrm{n}=$ densidad $\left(\right.$ ind. $\left.\mathrm{m}^{-2}\right) ; \mathrm{b}=$ biomasa $\left(\mathrm{g} \cdot \mathrm{m}^{-2}\right) ; \mathrm{S}=\operatorname{especies}\left(\mathrm{spp} .0,05 \mathrm{~m}^{-2}\right)$ : $\mathrm{H}^{\prime}=$ Diversidad (bitios. ind $^{-1}$ )

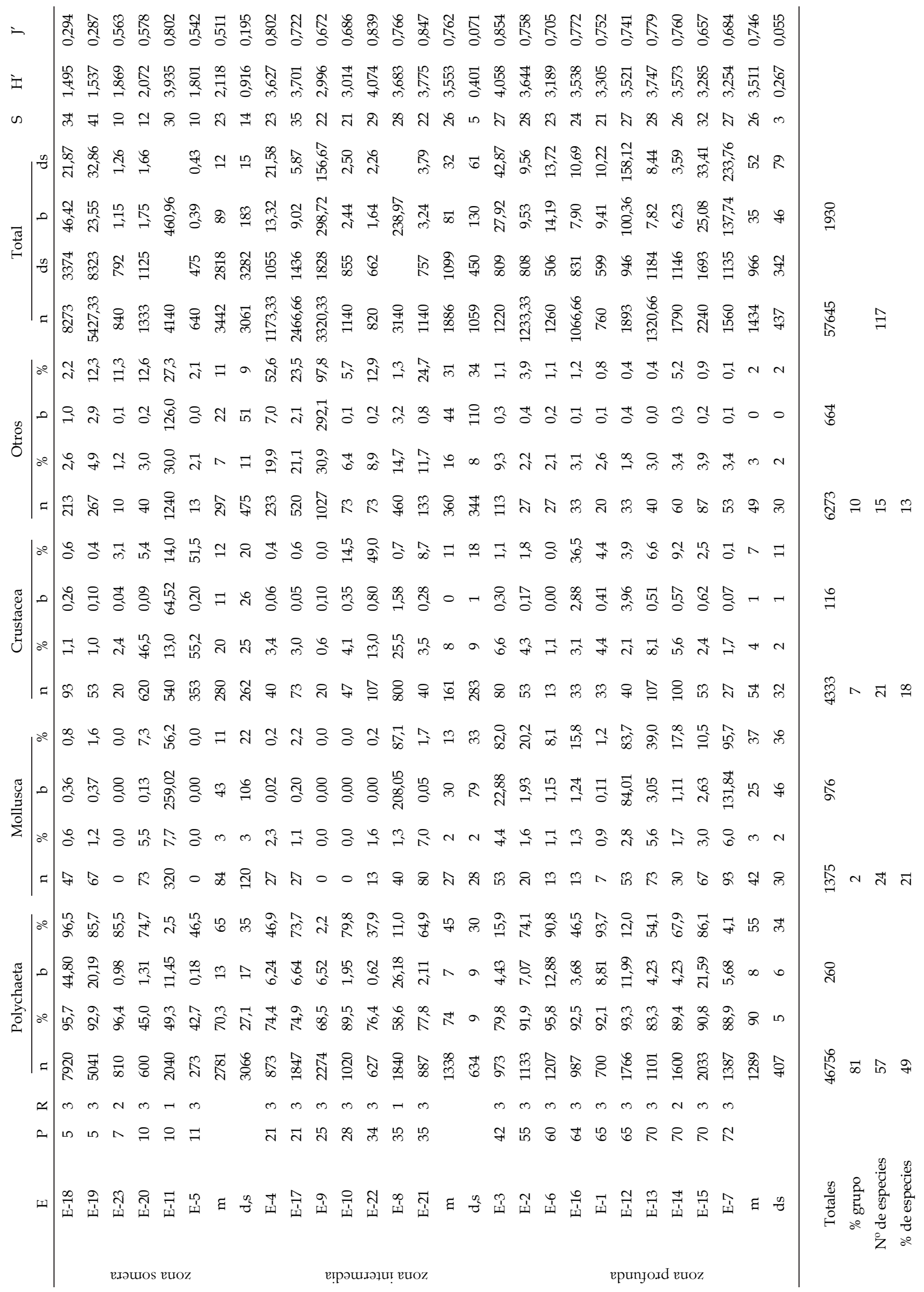


Tabla 3. Especies más importantes en cuanto al porcentaje de su densidad (a), frecuencia (b) de la macrofauna y los gremios tróficos (GT) de los poliquetos en la ensenada de Sechura, julio de 1998. Depositívoros Superficiales (SDF), Consumidores Superficiales (IF), Omnívoros/Depredadores (OP), Depositívoros Subsuperficiales (SSDF)

\begin{tabular}{|c|c|c|c|c|c|c|c|c|}
\hline & a) Densidad & & & & & b) Frecuencia & & \\
\hline $\mathrm{N}^{\circ}$ & Grupo & Familia & Especie & $\%$ & GT & Grupo & Especie & $\%$ \\
\hline 1 & Polychaeta & Spionidae & Polydora socialis & 23,39 & SDF & Polychaeta & Capitellidae & 86,96 \\
\hline 2 & Polychaeta & Spionidae & Paraprionospio pinnata & 12,50 & IF & Nemertea & Nemertinea III & 86,96 \\
\hline 3 & Polychaeta & Capitellidae & Capitellidae & 8,60 & SDF & Polychaeta & Paraprionospio pinnata & 82,61 \\
\hline 4 & Polychaeta & Spionidae & Prionospio peruana & 5,82 & IF & Polychaeta & Prionospio peruana & 82,61 \\
\hline 5 & $\begin{array}{l}\text { Echinoder- } \\
\text { mata }\end{array}$ & & Ophiuroidea & 4,18 & $\mathrm{OP}$ & Polychaeta & Spiophanes sp. & 78,00 \\
\hline 6 & Polychaeta & Paraonidae & Aricidea simplex & 3,73 & SDF & Polychaeta & Aricidea simplex & 73,91 \\
\hline 7 & Crustacea & & Oedicerotidae & 2,21 & IF & Polychaeta & Nereis sp. & 73,91 \\
\hline 8 & Sipunculida & & Sipunculidae & 2,10 & OP & Polychaeta & Aglaophamus dicirris & 65,22 \\
\hline 9 & Polychaeta & Nereidae & Nereis sp. & 2,04 & $\mathrm{OP}$ & Polychaeta & Glycera sp. & 65,22 \\
\hline 10 & Polychaeta & Spionidae & Spiophanes sp. & 1,99 & IF & Polychaeta & Parandalia fauveli & 65,22 \\
\hline 11 & Polychaeta & Ampharetidae & Ampharetidae & 1,98 & SDF & Mollusca & Tellina sp. & 65,22 \\
\hline 12 & Polychaeta & Spionidae & Minuspio sp. & 1,85 & IF & Polychaeta & Magelona phyllisae & 60,87 \\
\hline 13 & Polychaeta & Magelonidae & Magelona phyllisae & 1,74 & SDF & Polychaeta & Axiothella sp. & 60,87 \\
\hline 14 & Polychaeta & Lumbrineridae & Lumbrineris sp. & 1,73 & $\mathrm{OP}$ & Polychaeta & Minuspio sp. & 56,52 \\
\hline 15 & Nemertea & & Nemertinea III & 1,63 & OP & Echinodermata & Ophiuroidea & 56,52 \\
\hline 16 & Polychaeta & Hesionidae & Hesionidae & 1,48 & $\mathrm{OP}$ & Polychaeta & Hesionidae & 52,17 \\
\hline 17 & Crustacea & & Ostracoda & 1,42 & IF & Polychaeta & Harmothoinae & 52,17 \\
\hline 18 & Polychaeta & Syllidae & Syllidae & 1,11 & $\mathrm{OP}$ & Polychaeta & Ampharetidae & 47,83 \\
\hline 19 & Polychaeta & Onuphidae & Onuphis sp. & 1,08 & OP & Polychaeta & Lumbrineris sp. & 47,84 \\
\hline 20 & Polychaeta & Maldanidae & Axiothella sp. & 0,99 & SSDF & Crustacea & Oedicerotidae & 47,85 \\
\hline 21 & Mollusca & & Tellina sp & 0,91 & IF & Polychaeta & Sigambra tentaculata & 43,48 \\
\hline 22 & Polychaeta & Onuphidae & Aglaophamus dicirris & 0,90 & $\mathrm{OP}$ & Arthropoda & Cumacea & 43,48 \\
\hline 23 & Arthropoda & & Isopoda & 0,84 & $\mathrm{OP}$ & Polychaeta & Armandia sp. & 34,78 \\
\hline 24 & Porifera & & Porifera & 0,83 & SF & Polychaeta & Glycinde sp. & 34,78 \\
\hline 25 & Crustacea & & Crustacea2 & 0,77 & $\mathrm{OP}$ & Sipunculida & Sipunculidae & 34,78 \\
\hline 26 & Polychaeta & Glyceridae & Glycera sp. & 0,73 & OP & Polychaeta & Polydora socialis & 30,43 \\
\hline 27 & Polychaeta & Spionidae & Scolelepis sp. & 0,73 & IF & Polychaeta & Onuphis sp. & 30,43 \\
\hline 28 & Polychaeta & Cirratulidae & Cirratulidae & 0,71 & SDF & Polychaeta & Phyllodoce sp. & 30,43 \\
\hline 29 & Polychaeta & Polynoidae & Harmothoinae & 0,64 & $\mathrm{OP}$ & Polychaeta & Scolelepis sp. & 30,43 \\
\hline 30 & Polychaeta & Phyllodocidae & Phyllodocesp. & 0,62 & $\mathrm{OP}$ & Crustacea & Crustacea2 & 30,43 \\
\hline
\end{tabular}

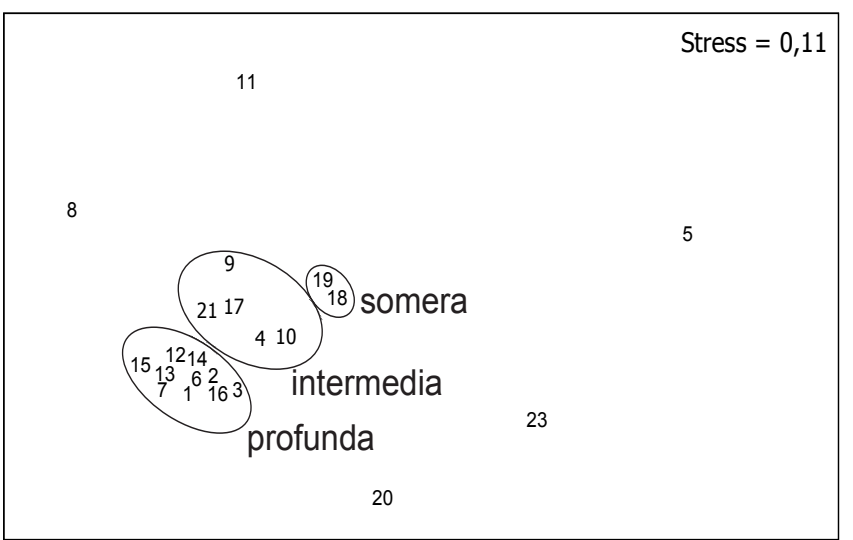

Figura 2. Análisis de Escalamiento no métrico multidimensional de la macrofauna bentónica en la Ensenada de Sechura, julio de 1998.

\section{Parámetros comunitarios de la comunidad macro- zoobentónica}

Fueron registradas en total 117 especies o grupos taxonómicos de la macrofauna distribuidas en 4 grandes grupos: 57 Polychaeta, 24 Crustacea, 21 Mollusca y 15 clasificados como 'Otros', el cual agrupa a Echinodermata, Nemertinea, Sipunculida, Porifera, Actiniaria, Platyhelminthes, Echiurida, Priapulida, Olygochaeta, Hemichordata. El grupo Polychaeta fue el mejor representado con el $49 \%$ del total de especies (Tabla 2).

El rango del número promedio de especies por estación varió de 10 spp. por $0,05 \mathrm{~m}^{-2}$ (E-5, $11 \mathrm{~m}$ y E-23, $7 \mathrm{~m}$ ) a $41 \mathrm{spp}$. por $0,05 \mathrm{~m}^{-2}(\mathrm{E}-19,5 \mathrm{~m})$ (Tabla 2). En las estaciones costeras de la parte sur se registraron los mayores valores.

La densidad media de la macrofauna estuvo entre $640 \pm 475$ ind. $\mathrm{m}^{-2}(\mathrm{E}-5,11 \mathrm{~m})$ y $8273 \pm 3374$ ind. $\mathrm{m}^{-2}(\mathrm{E}-18,5 \mathrm{~m}$ ) (Tabla 
Tabla 4. Análisis de Correlación Simple entre los parámetros físico, quimico, sedimentlógicos y biológicos en la ensenada de Sechura, julio de 1998. (Prof= profundidad; T=temperatura; OD = oxígeno disuelto; \%MOT = matería orgánica total; $\mathrm{nPo}=$ densidad Polychaeta; $\mathrm{nMo}=$ densidad Mollusca; $\mathrm{nCr}=$ densidad Crustacea; $\mathrm{nEQ}=$ densidad Echinodermata; $\mathrm{nNE}=$ densidad Nemertea; $\mathrm{nOT}=$ densidad otros; $\mathrm{nTO}=$ densidad total; $\mathrm{bTO}=$ biomasa total; $\mathrm{sPo}=$ especies Polychaeta; $\mathrm{sMo}=$ especies $\mathrm{Mollusca}$; $\mathrm{sCr}=$ especie Crustacea; $\mathrm{sNE}=$ especie Nemertea; $\mathrm{sEQ}=$ especie Echinodermata; $\mathrm{sOT}=$ especie otros; $\mathrm{sTO}=$ especie total; $\mathrm{H}^{\prime}$ = Diversidad; J'= equidad; \% IF= consumidores superficiales; \%OP= omnívoros/depredadores; \%SDF= depositívoros superficiales; $\%$ SSDF= depositívoros subsuperficiales; \%SF= suspensívoros). Las correlaciones marcadas son significantes a $p<0,05$.

\begin{tabular}{|c|c|c|c|c|c|c|c|c|c|c|c|c|c|c|c|}
\hline & Prof & $\mathrm{T}$ & OD & \%MOT & $\%$ Are & $\% \mathrm{Li}$ & $\% \mathrm{Ar}$ & nPo & nMo & $\mathrm{nCr}$ & nEQ & $\mathrm{nNE}$ & nOt & nTo & \\
\hline Prof & 1.00 & & & & & & & & & & & & & & \\
\hline $\mathrm{T}$ & -0.86 & 1.00 & & & & & & & & & & & & & \\
\hline OD & -0.50 & 0.42 & 1.00 & & & & & & & & & & & & \\
\hline \%МOT & -0.32 & 0.19 & 0.15 & 1.00 & & & & & & & & & & & \\
\hline \%Arena & -0.38 & 0.09 & 0.03 & 0.02 & 1.00 & & & & & & & & & & \\
\hline$\%$ Limo & 0.35 & -0.04 & -0.05 & -0.16 & -0.98 & 1.00 & & & & & & & & & \\
\hline \%Arcilla & 0.42 & -0.42 & -0.24 & 0.41 & -0.52 & 0.40 & 1.00 & & & & & & & & \\
\hline nPo & -0.33 & 0.24 & 0.68 & -0.07 & 0.17 & -0.15 & -0.23 & 1.00 & & & & & & & \\
\hline nMo & -0.14 & 0.10 & 0.32 & -0.24 & 0.20 & -0.21 & 0.09 & 0.14 & 1.00 & & & & & & \\
\hline $\mathrm{nCr}$ & -0.34 & 0.28 & 0.22 & 0.07 & 0.32 & -0.38 & -0.41 & -0.07 & 0.41 & 1.00 & & & & & \\
\hline nEQ & -0.29 & 0.33 & 0.50 & 0.11 & -0.40 & 0.40 & 0.17 & 0.08 & 0.67 & 0.28 & 1.00 & & & & \\
\hline $\mathrm{nNE}$ & -0.34 & 0.24 & 0.60 & 0.13 & -0.07 & 0.08 & 0.10 & 0.73 & -0.09 & -0.23 & -0.01 & 1.00 & & & \\
\hline nOt & -0.32 & 0.18 & 0.43 & 0.62 & 0.01 & -0.13 & 0.04 & 0.17 & 0.13 & 0.40 & 0.32 & 0.15 & 1.00 & & \\
\hline nTo & -0.43 & 0.32 & 0.78 & -0.00 & 0.17 & -0.16 & -0.25 & 0.96 & 0.32 & 0.13 & 0.30 & 0.68 & 0.33 & 1.00 & \\
\hline bTo & -0.17 & 0.22 & 0.55 & 0.09 & -0.32 & 0.25 & 0.08 & 0.15 & 0.67 & 0.46 & 0.88 & -0.05 & 0.47 & 0.38 & \\
\hline sPo & 0.42 & -0.53 & 0.07 & -0.02 & -0.24 & 0.18 & 0.29 & 0.39 & -0.19 & -0.22 & -0.39 & 0.49 & 0.17 & 0.30 & \\
\hline sMo & -0.06 & -0.03 & 0.38 & -0.20 & 0.11 & -0.10 & -0.09 & 0.55 & 0.25 & -0.05 & -0.16 & 0.65 & 0.06 & 0.51 & \\
\hline $\mathrm{sCr}$ & -0.13 & 0.07 & 0.04 & 0.06 & 0.26 & -0.25 & -0.14 & 0.30 & -0.12 & 0.07 & -0.31 & 0.31 & -0.09 & 0.24 & \\
\hline sEQ & -0.39 & 0.42 & 0.29 & 0.47 & 0.09 & -0.14 & 0.14 & -0.07 & 0.41 & 0.23 & 0.49 & -0.01 & 0.47 & 0.08 & \\
\hline sNE & -0.04 & -0.09 & 0.21 & 0.10 & 0.03 & -0.06 & 0.29 & 0.60 & -0.33 & -0.39 & -0.21 & 0.63 & -0.05 & 0.48 & \\
\hline sOt & -0.43 & 0.37 & 0.50 & 0.55 & 0.20 & -0.24 & 0.13 & 0.26 & 0.46 & 0.15 & 0.59 & 0.29 & 0.56 & 0.40 & \\
\hline sTO & 0.13 & -0.28 & 0.19 & 0.04 & 0.01 & -0.04 & 0.16 & 0.51 & -0.12 & -0.12 & -0.36 & 0.65 & 0.16 & 0.43 & \\
\hline $\mathbf{H}^{\prime}$ & 0.56 & -0.45 & -0.42 & 0.25 & -0.43 & 0.33 & 0.61 & -0.49 & 0.21 & -0.05 & 0.17 & -0.47 & 0.22 & -0.42 & \\
\hline $\mathrm{J}^{\prime}$ & 0.51 & -0.38 & -0.59 & 0.21 & -0.43 & 0.33 & 0.58 & -0.73 & 0.11 & 0.01 & 0.14 & -0.66 & 0.10 & -0.66 & \\
\hline$\%$ IF & 0.46 & -0.52 & -0.44 & -0.58 & -0.07 & 0.12 & -0.05 & -0.39 & -0.24 & -0.20 & -0.28 & -0.31 & -0.53 & -0.48 & \\
\hline$\% \mathrm{OP}$ & -0.20 & 0.40 & -0.05 & 0.27 & 0.15 & -0.15 & -0.34 & -0.34 & 0.24 & 0.56 & 0.28 & -0.47 & 0.25 & -0.19 & \\
\hline$\%$ SDF & -0.38 & 0.26 & 0.50 & 0.42 & -0.01 & -0.05 & 0.31 & 0.69 & 0.01 & -0.27 & 0.05 & 0.73 & 0.34 & 0.65 & \\
\hline$\%$ SF & -0.24 & 0.25 & 0.45 & 0.25 & 0.02 & -0.16 & -0.12 & 0.06 & 0.82 & 0.65 & 0.73 & -0.23 & 0.41 & 0.29 & \\
\hline \multirow[t]{2}{*}{$\%$ SSDF } & 0.62 & -0.40 & -0.32 & -0.14 & -0.40 & 0.35 & 0.17 & -0.22 & 0.04 & 0.03 & -0.20 & -0.28 & -0.02 & -0.24 & \\
\hline & bTo & sPo & sMo & $\mathrm{sCr}$ & sEQ & sNE & $\mathrm{sOt}$ & sTO & $\mathrm{H}^{\prime}$ & $\mathrm{J}^{\prime}$ & $\%$ IF & $\% \mathrm{OP}$ & $\%$ SDF & $\% \mathrm{SF}$ & $\%$ SSDF \\
\hline bTo & 1.00 & & & & & & & & & & & & & & \\
\hline sPo & -0.24 & 1.00 & & & & & & & & & & & & & \\
\hline sMo & -0.04 & 0.61 & 1.00 & & & & & & & & & & & & \\
\hline sCr & -0.32 & 0.38 & 0.42 & 1.00 & & & & & & & & & & & \\
\hline sEQ & 0.39 & -0.29 & -0.03 & -0.04 & 1.00 & & & & & & & & & & \\
\hline sNE & -0.23 & 0.39 & 0.16 & 0.30 & -0.30 & 1.00 & & & & & & & & & \\
\hline sOt & 0.51 & -0.14 & 0.18 & 0.06 & 0.77 & -0.06 & 1.00 & & & & & & & & \\
\hline sTO & -0.26 & 0.90 & 0.77 & 0.66 & -0.12 & 0.45 & 0.06 & 1.00 & & & & & & & \\
\hline $\mathbf{H}^{\prime}$ & 0.20 & 0.14 & -0.20 & -0.04 & 0.29 & -0.27 & 0.11 & -0.01 & 1.00 & & & & & & \\
\hline $\mathrm{J}^{\prime}$ & 0.13 & -0.11 & -0.45 & -0.19 & 0.24 & -0.38 & -0.04 & -0.28 & 0.94 & 1.00 & & & & & \\
\hline$\%$ IF & -0.29 & 0.05 & -0.18 & -0.10 & -0.64 & -0.08 & -0.62 & -0.09 & -0.07 & 0.08 & 1.00 & & & & \\
\hline$\% \mathrm{OP}$ & 0.32 & -0.51 & -0.30 & -0.19 & 0.47 & -0.69 & 0.28 & -0.49 & 0.18 & 0.26 & -0.46 & 1.00 & & & \\
\hline$\%$ SDF & -0.01 & 0.33 & 0.40 & 0.26 & 0.30 & 0.66 & 0.44 & 0.47 & -0.13 & -0.35 & -0.69 & -0.32 & 1.00 & & \\
\hline$\% \mathrm{SF}$ & 0.83 & -0.32 & -0.03 & -0.21 & 0.46 & -0.40 & 0.47 & -0.31 & 0.25 & 0.19 & -0.41 & 0.49 & -0.02 & 1.00 & \\
\hline$\%$ SSDF & 0.08 & 0.35 & 0.13 & -0.05 & -0.23 & -0.19 & -0.31 & 0.16 & 0.50 & 0.43 & 0.18 & 0.01 & -0.30 & 0.05 & \\
\hline
\end{tabular}


2). Las mayores densidades se registraron frente a Parachique. El grupo taxonómico con mayor densidad total fue Polychaeta con $81 \%$ (Tabla 2). Dentro de los poliquetos, Polydora socialis (Schmarda, 1861) fue la especie dominante con 23\% (Tabla 3a). La biomasa varió entre $0,4 \pm 0,43$ g.m $\mathrm{m}^{-2}(\mathrm{E}-5,11 \mathrm{~m})$ y 461 g.m $\mathrm{m}^{-2}(\mathrm{E}-11,10 \mathrm{~m})$ (Tabla 2).

Los taxa más frecuentes fueron los poliquetos de la familia Capitellidae (87\%), Paraprionospio pinnata (Ehlers, 1901) y Prionospio peruana (Hartmann-Schroder, 1962) (83\%), Spiophanes sp. (78\%), Aricidea simplex (Day, 1963) y Nereis sp. (74\%) y Nemertinea III (87\%) (Tabla 3b).

La distribución de la densidad por estratos mostró tendencia a la disminución desde las estaciones someras hacia las más profundas; el grupo Polychaeta fue el dominante en abundancia (entre 49 y 96\%), en todas las estaciones excepto en E-20 y E-5 (Tabla 2).

Los indicadores cuantitativos de la estructura comunitaria mostraron un amplio rango de variación. El índice de diversidad de Shannon y Wiener (H') varió entre 1,4 (E-18, 5 m) y 3,9 bitios.ind. ${ }^{-1}$ (E-11, $10 \mathrm{~m}$ ) y el índice de Equidad de Pielou (J') estuvo comprendido entre $0,33-0,89$; hallándose los mayores valores en la zona intermedia y profunda (Tabla 2).

El análisis NMDS de clasificación multivariada entre estaciones permitió diferenciar la formación de 3 grupos, el cual muestra que las 23 estaciones de muestreo se agrupan de acuerdo a la profundidad en base a su distancia en el mapa con un estrés de 0,1 que implica una buena representación (Fig. 2). El primer grupo estuvo formado por las estaciones someras E-18 y E-19, (estaciones costeras de textura arenosa y rocosa) de $5 \mathrm{~m}$ de profundidad; las densidades y número de especies fueron altos, influenciadas posiblemente por encontrarse frente al estuario de Virrilá. La dominancia en abundancia fue del poliqueto depositívoro superficial $P$. socialis (54\%) (Tabla 6).

El segundo grupo estuvo formado por las estaciones E-4, E-9, E-10, E-17, E-21 y E-22, las cuales se encuentran entre 21 y $35 \mathrm{~m}$ de profundidad. Texturalmente el tipo de sustrato dominante en este grupo fue arena limosa y se caracterizó por la presencia dominante de poliquetos $(>70 \%)$ (Tabla 2) de la familia Capitellidae (17\%) y las especies $P$. pinnata y $A$. simplex (11\%) (Tabla 6).

El tercer grupo estuvo formado por las estaciones E-1, E2, E-3, E-6, E-7, E-12, E-13, E-14, E-15 y E-16, ubicadas a profundidades entre 42 y $72 \mathrm{~m}$, cuyo tipo de sedimento predominante fue arena y limo arenoso (Tabla 1). La dominancia de los poliquetos se incrementó en este grupo (>80\%) (Tabla 2), principalmente por las especies $P$. pinnata $(27 \%)$ y $P$. peruana (14\%) (Tabla 6).

De otro lado, distribuidas sin mostrar afinidad a ningún grupo se hallaron las estaciones E-23, E-20, E-11, E-8 y E-5. Salvo la estación 8 , ubicada a $35 \mathrm{~m}$ de profundidad sobre sustrato de arena y grava, se trata de estaciones someras $(7-11 \mathrm{~m})$ sobre arena y roca. Los valores de densidad y número de especies resultaron menores a las estaciones E-18 y E-19, (donde dominó P. socialis), presentándose dominancia de Spiophanes sp. en E-23, Ostracoda y $P$. pinnata en E-20, Ophiuroidea y Syllidae en E-11, Isopoda y Hemipodus sp. en E-5 y de Capitellidae y Oedicerotidae en E-8.

\section{Relaciones entre los factores abióticos y la estruc- tura comunitaria}

La profundidad en la Ensenada de Sechura tuvo correlaciones significativas con varios parámetros comunitarios, influenciando en el número de poliquetos $(\mathrm{r}=0,42 ; \mathrm{p}=0,44)$, la diversidad $(\mathrm{r}=$ $0,56 ; \mathrm{p}=0,005)$ y la equidad total $(\mathrm{r}=0,51 ; \mathrm{p}=0,13)$, mientras que el oxígeno con la densidad y biomasa total $(\mathrm{r}=0,78, \mathrm{p}=$ 0,$001 ; \mathrm{r}=0,55, \mathrm{p}=0,007$ respectivamente); especialmente con la densidad de poliquetos $(\mathrm{r}=0,67, \mathrm{p}=0,001)$, equinodermos $(\mathrm{r}=$ $0,50, \mathrm{p}=0,001$, menos tolerantes a la hipoxia) y nemertinos $(\mathrm{r}=$ $0,60, \mathrm{p}=0,003$, depredadores) (Tabla 4).

En el Análisis de Correspondencia Canónica (ACC) (Tabla 3 ), las variables que se correlacionaron significativamente con el primer eje fueron profundidad $(r=0,89)$, temperatura $(r=-0,85)$, arcilla $(r=0,70)$, limo $(r=0,63)$ y oxígeno $(r=-0,58$. En tanto la única variable que se correlacionó significativamente con el segundo eje fue la materia orgánica $(\mathrm{r}=0,56)$ (Fig. 3).

El primer eje explicó el 29,4\% de la variabilidad total de los datos de las taxa, mientras que el segundo eje explicó una variabilidad del 11,2\%. Por lo tanto los primeros dos ejes juntos explicaron el 40,6\% de la varianza en la correlación de los taxa respecto a las variables ambientales. Los ejes restantes solamente contribuyeron marginalmente con menos del $8 \%$ de la varianza cada uno, por lo que la interpretación de los resultados se basa en los dos primeros ejes (Fig. 3).

La ordenación respecto al Eje 1, muestra que $P$. pinnata, $P$. peruana, Ampharetidae, Minuspio sp., Lumbrineris sp., Nemertinea III, Capitellidae y $A$. simplex se ubicaron mayormente en estaciones más profundas, asociadas a sedimentos arcillosos y limosos. En tanto que, Nereis sp., Hesionidae, Ophiuroidea, Oedicerotidae, M. phyllisae, Sipunculidae, Spiophanes y P. socialis se encontraron asociadas a ambientes cálidos y oxigenados, como fueron las estaciones arenosas menos profundas.

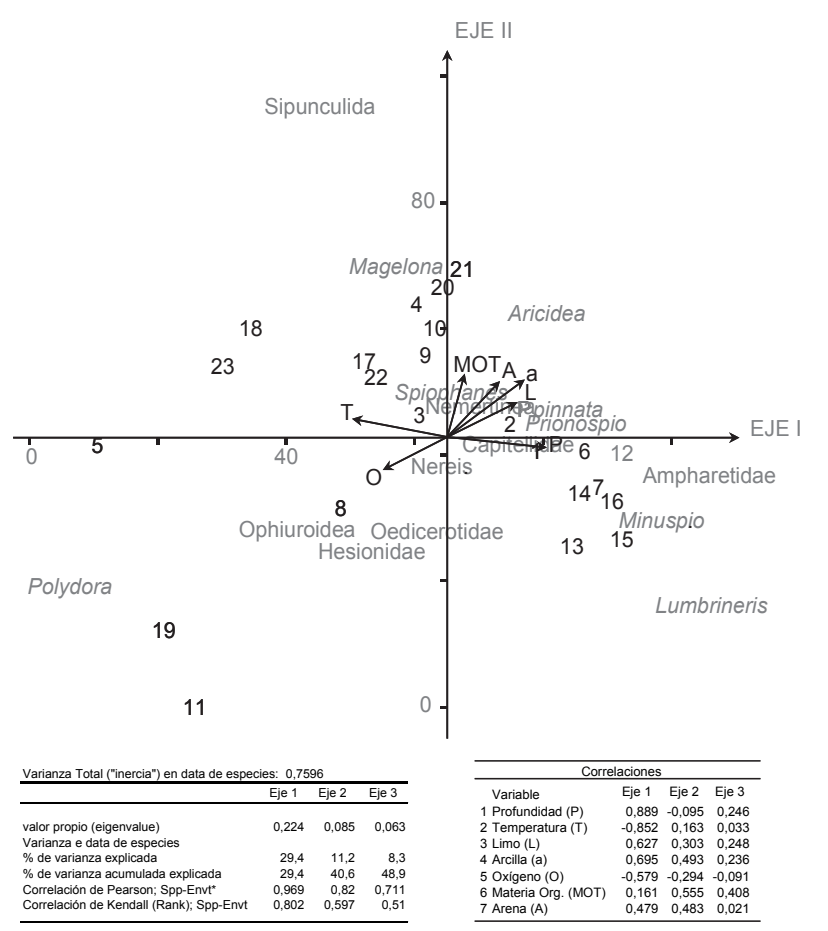

Figura 3. Análisis de Correspondencia canónica entre las estaciones, especies más representativas, profundidad, temperatura, limo y arcilla en la Ensenada de Sechura, julio de 1998. 
Tabla 5. Porcentaje de la densidad de los gremios tróficos de los poliquetos en base a la textura sedimentaria en la Ensenada de Sechura, julio 1998.

\begin{tabular}{|c|c|c|c|c|c|c|}
\hline \% Gremio trófico & & arena & $\begin{array}{c}\text { arena } \\
\text { limosa }\end{array}$ & $\begin{array}{c}\text { limo } \\
\text { arenoso }\end{array}$ & $\begin{array}{c}\text { arena } \\
\text { arcillosa }\end{array}$ & roca \\
\hline consumidores superficiales & (IF) & 47,30 & 20,62 & 25,79 & 3,58 & 2,71 \\
\hline depositívoros superficiales & $(\mathrm{SDF})$ & 43,78 & 14,94 & 10,09 & 5,18 & 26,01 \\
\hline depositívoros subsuperficiales & (SSDF) & 20,00 & 28,84 & 23,26 & 20,47 & 7,44 \\
\hline suspensívoros & $(\mathrm{SF})$ & 0,00 & 3,13 & 0,00 & 28,13 & 68,75 \\
\hline omnívoros/depredadores & $(\mathrm{OP})$ & 25,77 & 20,17 & 17,44 & 15,88 & 20,75 \\
\hline
\end{tabular}

Con respecto al Eje 2 se encontró a Capitellidae, $A$. simplex, Sipunculida, $M$. phyllisae asociadas a estaciones con mayor porcentaje de MOT en el sedimento contrariamente a Nereis sp. y Oedicerotidae.

En cuanto a los gremios tróficos del grupo Polychaeta, los depositívoros superficiales (SDF) fueron el grupo más abundante, contribuyendo en un $50 \%$ de la densidad en toda la ensenada. $\mathrm{Su}$ distribución fue mayor en fondos de arena y roca (Tabla 5), exhibiendo además una correlación positiva con el oxígeno de fondo $(r=0,50 ; p=0,014)$ (Tabla 4).

Seguidamente los consumidores superficiales (IF) obtuvieron un $29 \%$ de la abundancia total de poliquetos, principalmente en texturas de arena y limo. El porcentaje de IF mostró una correlación positiva con la profundidad $(r=0,46 ; p=0,028)$ y correlaciones negativas con la temperatura, oxígeno y el porcentaje de MOT $(r=-0,52 ; \mathrm{p}=0,011 ; \mathrm{r}=-0,44 ; \mathrm{p}=0,035 ; \mathrm{r}=-0,58$; $\mathrm{p}=0,006$; respectivamente). Los depositívoros subsuperficiales (SSDF) alcanzaron su mayor abundancia relativa en texturas de arena limosa; el porcentaje de SSDF exhibió una correlación positiva con la profundidad $(r=0,62 ; \mathrm{p}=0,020)$. En cambio los suspensívoros (SF) se encontraron mayormente en fondo rocoso y el porcentaje de SF presentó una correlación positiva con el oxígeno disuelto $(r=0,45 ; \mathrm{p}=0,031)$. Los omnívoros/depredadores (OP) totalizaron un $20 \%$ de la abundancia de poliquetos, presentándose en todas las texturas.

\section{Discusión}

\section{Comparación con otras bahías del litoral peruano.}

A diferencia de otras bahías de similar profundidad, la ensenada de Sechura está ubicada en la zona de convergencia de aguas frías de la Corriente Costera Peruana (CCP) y de aguas cálidas ecuatoriales superficiales. Además se caracteriza por mostrar una distribución de sedimentos heterogénea, con predominancia de arena, arena limosa y limo arenoso.

Estas condiciones de mezcla de masas de agua y de distribución de sedimentos probablemente expliquen los valores relativamente elevados de la biomasa y la diversidad en relación a otras bahías

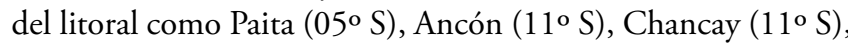
Independencia $\left(15^{\circ} \mathrm{S}\right)$ e Ilo $\left(17^{\circ} \mathrm{S}\right)$ (cuyos valores fueron uniformados a ind. $\mathrm{m}^{2}$ y bitios.ind ${ }^{-1} \log _{\mathrm{n}}$ en la Tabla 6). En términos de biomasa, convertida a unidades de peso seco libre de ceniza, los valores obtenidos en el presente estudio $\left(0,06-126,81\right.$ g.m $\left.{ }^{-2}\right)$ tienden a superar en un orden de magnitud a los de las bahías de la costa centro (Ancón y Chancay) y sur (Independencia e Ilo), aunque son comparables a los valores de biomasa en la bahía de Paita (L. Quipúzcoa com. pers.). En densidad, los valores alcanzados se encuentran aproximadamente en el mismo rango reportado para la bahía de Paita a profundidades someras e intermedias, mientras que los valores máximos alcanzados son inferiores en un factor de al menos $100 \%$ respecto a las bahías del sur (Tarazona et al. 1990; L. Quipúzcoa com. pers.). Finalmente, aunque en periodos diferentes las comunidades de Sechura presentan un rango de riqueza y de diversidad H' mayor que el resto de las bahías de la costa y especialmente aquellas de la costa central (Tabla 6).

Con excepción de Ancón, los taxa dominantes encontrados en el presente estudio tienen en común con otras bahías a poliquetos de las familias Capitellidae o Spionidae (Tabla 6). Estos organismos suelen tener un comportamiento depositívoro subsuperficial o depositívoro superficial, respectivamente (Wheatcroft et al., 1994; Gutiérrez, 2000).

\section{Influencia del contexto oceanográfico}

El presente estudio se desarrolló en la fase final de El Niño 1997-98, considerado uno de los eventos más fuertes ocurridos sobre el Pacífico Ecuatorial Central y Oriental en los últimos 150 años (Jaimes, 1999). Diversos estudios han abordado los impactos de EN sobre las comunidades bentónicas frente a la costa peruana. Frente a la costa central, en la bahía de Ancón, la abundancia, biomasa y diversidad aumentaron durante EN 1997-1998 (al igual que durante EN 1982-83) debido a la oxigenación de la capa subsuperficial (Tarazona et al., 1988; 1996; 2000). En cambio, en la zona norte frente a Paita $\left(05^{\circ} S\right)$ y a San José $\left(07^{\circ} S\right)$ los valores de abundancia, biomasa y riqueza se redujeron significativamente durante EN 1997-1998, a diferencia de la respuesta de la macrofauna bentónica frente a la costa central. Este comportamiento frente a Paita pudo obedecer a los elevados aportes de sedimento producidos por las descargas del río Chíra, mientras que frente a San José, pudo resultar por la disminución local de la producción primaria y por lo tanto del flujo de alimento partículado al bentos (Gutiérrez et al., 2005). En la bahía de Ilo la abundancia y biomasa disminuyeron durante EN 1997-1998, paralelamente al incremento de los valores de la temperatura y del contenido de oxígeno cerca del fondo, aumentando nuevamente después de EN (L. Quipúzcoa com. pers.).

En la Tabla 6, se comparan los parámetros comunitarios en la ensenada de Sechura al inicio de EN 1997-98 (L. Quipúzcoa, com. pers.) con los resultados, obtenidos a fines de EN 199798. Las principales diferencias son los valores más elevados de biomasa y de riqueza específica. Sin embargo con la prueba de U-Mann-Whitney (Tabla 7) no se encontraron diferencias significativas ( $p>0,05)$ entre ambos períodos para las variables de densidad, biomasa, número de especies, diversidad y equidad.

En cuanto a la composición de las especies dominantes, a inicio de EN 1997-98, el poliqueto consumidor de interfase Owenia sp. y el poliqueto depositívoro subsuperficial Mediomastus 
Tabla 6. Promedio y rango de los parámetros comunitarios de la macrofauna en diferentes bahías del litoral peruano. $p=$ rango de profundidad $(m) ; N=n^{\circ}$ de estaciones; $b=$ biomasa (peso seco sin cenizas g. $\left.m^{-2}\right) ; n=$ densidad (ind $\left.\cdot m^{-2}\right) ; S=n^{\circ}$ especies $\left({ }^{*} n^{\circ}\right.$ spp. 0,2 m-2; ** $n^{\circ}$ spp. 0,05 m²); $\mathrm{H}^{\prime}=$ diversidad Shannon (bitios.ind ${ }^{-1}$ ), base =log n. " $\mathrm{n}$ " fue transformada a ind. $\mathrm{m}^{-2} ;$ a excepción de Ancón e Independencia, la biomasa en el resto de bahías fue transformada de peso húmedo a peso seco sin cenizas g. $\mathrm{m}^{-2}$.

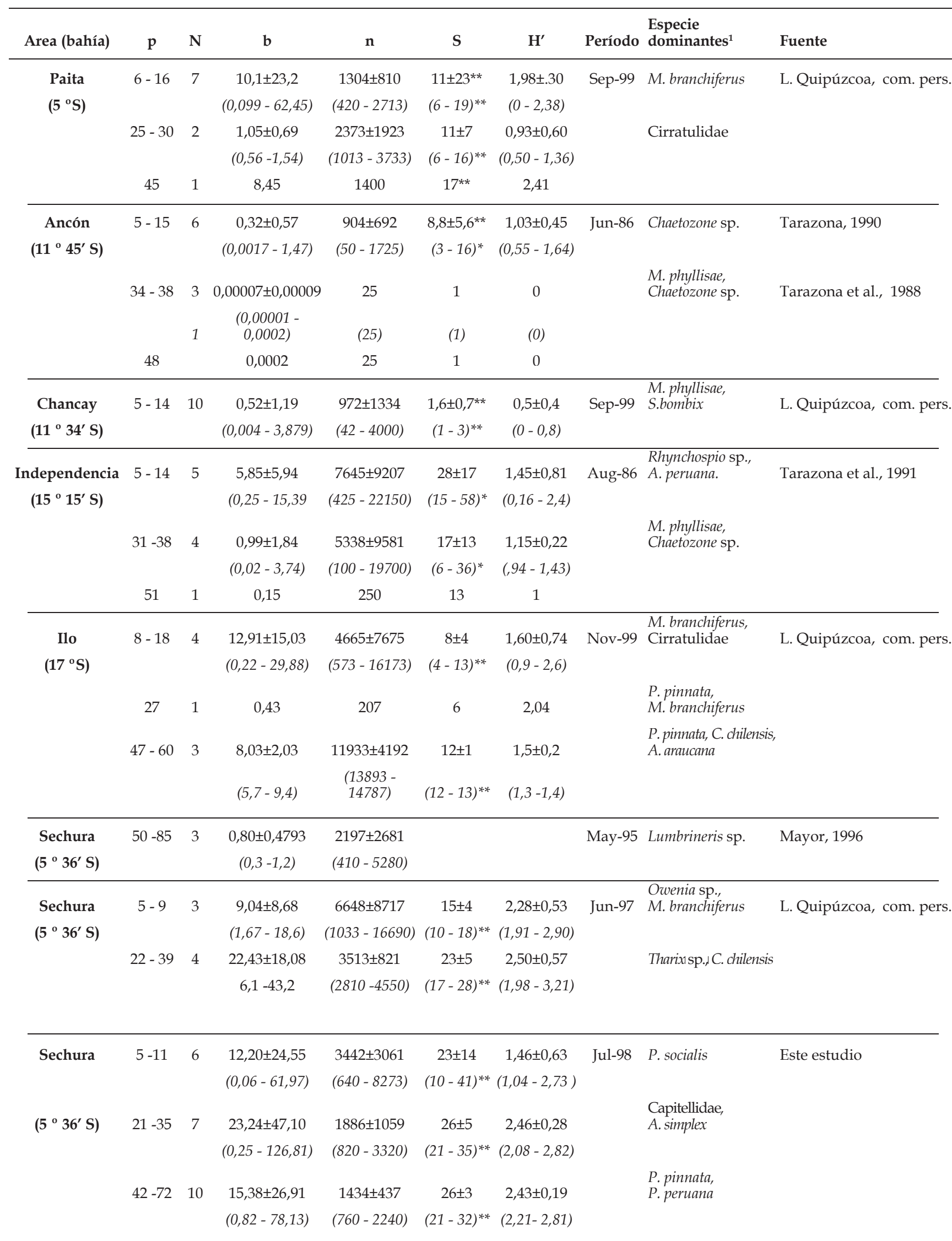

${ }^{1}$ Mediomastus branchiferus, Magelona phyllisae, Spiophanes bombix, Paraprionospio pinnata, Cossura chilensis, Ampelisca arucana, Aricidia simplex, Polydora socialis, Aquilaspio peruana, 
Tabla 7. Análisis de varianza de Kruskal-Wallis y grupos homogeneos $(p<0,05)$ según la prueba de U-Mann Whitney, considerando como variable independiente los grupos de estratos (I, II, III) y como variables dependientes a los parámetros comunitarios del macrobentos en la Ensenada de Sechura, julio 1998. $\mathrm{N}=$ densidad, $\mathrm{B}=$ biomasa, $\mathrm{S}=\mathrm{N}^{\circ}$ de especies, $\mathrm{J}$ = Indice de equidad, $\mathrm{H}^{\prime}$ = Indice de Shannon, nPo = abundancia de poliquetos, $\mathrm{nCru}=$ abundancia de crustáceos, $\mathrm{nMol}=$ abundancia de Moluscos, nOTr = Abundancia de otros grupos, $\mathrm{X}^{2}=$ Chi cuadrado; $\mathrm{gL}=$ grados de libertad; $\mathrm{p}=$ probabilidad Chicuadrado; $\mathrm{H}=$ test de Kruskal-Wallis; $\mathrm{p}_{\mathrm{k}-\mathrm{w}}=$ probalidad $\mathrm{K}-\mathrm{W}$.

\begin{tabular}{|c|c|c|c|c|c|c|}
\hline & $\chi^{2}$ & $g L$ & $\rho$ & $K-W$ & $\mathrm{p}_{k-w}$ & $\begin{array}{l}\text { Grupos } \\
\text { homogeneos }\end{array}$ \\
\hline $\mathrm{N}$ & 1.1683 & 2 & 0.5576 & 0.7261 & 0.6955 & \\
\hline B & 0.0996 & 2 & 0.9514 & 0.7106 & 0.7010 & \\
\hline S & 0.6884 & 2 & 0.7088 & 0.0999 & 0.9512 & \\
\hline $\mathrm{J}^{\prime}$ & 3.1720 & 2 & 0.2047 & 6.8862 & 0.0320 & $\underline{\mathrm{I}} \mathrm{III} \quad \mathrm{II}$ \\
\hline $\mathrm{H}^{\prime}$ & 3.9163 & 2 & 0.1411 & 6.6248 & 0.0364 & $\underline{I}$ \\
\hline nPol & 0.0996 & 2 & 0.9514 & 0.0182 & 0.9909 & \\
\hline nCru & 2.0532 & 2 & 0.3582 & 2.7413 & 0.2539 & \\
\hline nMol & 1.9125 & 2 & 0.3843 & 1.3152 & 0.5181 & \\
\hline nOTr & 13.3818 & 2 & 0.0012 & 11.5882 & 0.0030 & I $\quad$ II \\
\hline
\end{tabular}

branchiferus (Hartman, 1944) eran las especies dominantes en fondos arenosos someros, a diferencia de lo encontrado al final de EN 1997-98, donde tendió a dominar el poliqueto depositívoro superficial Polydora socialis. Sin embargo, dada la heterogeneidad del estrato somero, es difícil aseverar la ocurrencia de una variación temporal. Pero los cambios en la composición de las especies dominantes como Tharyx sp. y Cossura chilensis a inicios de EN 1997-98 encontradas en el sustrato intermedio pueden considerarse significativos, dada la homogeneidad de la composición a finales de EN 1997-98 detectada en este estudio (Tabla 6). Finalmente, en fondos más profundos, el único antecedente es dado por Mayor (1996), quien reportó a Lumbrineris sp. (gremio omnívoro/depredador según Gutiérrez et al., 2000) como especie dominante en mayo de 1995, mientras que en este estudio no lo fue.

\section{Zonación, sedimentos y gremios tróficos}

$\mathrm{Al}$ igual que otras bahías del litoral, la abundancia y la biomasa exhibieron una reducción de sus valores con la profundidad $(5$ a 72 m) (Tarazona, 1990; Tarazona et al., 1996; L. Quipuzcoa com. pers.). Debe tomarse en cuenta que tanto la temperatura como el oxigeno de fondo presentaron correlaciones significativas con la profundidad (Tabla 4). De ello se infiere que el patrón batimétrico de los parámetros comunitarios resulta de la combinación de varios factores abióticos asociados a la profundidad. Sin embargo la densidad, biomasa, número de especies, densidad de poliquetos, densidad de crustáceos y densidad de moluscos no mostraron diferencias significativas (ANOVA de Kruskal Wallis, p> 0,05) respecto a los estratos batimétricos. En cambio la equidad y la diversidad resultaron ser significativamente menores en el estrato somero respecto a los estratos intermedio y profundo (Tabla 7).

Las mayores densidades alcanzadas en las estaciones someras se deben principalmente al mayor aporte de las estaciones E-18 y E-19 (Figura 2), que se encuentran cerca a la margen derecha del estuario de Virrilá, la cual es una fuente ocasional de agua dulce en épocas de fuertes lluvias y crecientes del río Piura $(\mathrm{Hi}-$ dronav-34, 1995), como durante EN 1997-98. Frente a Paita,
Gutiérrez et al. (2005) determinaron que las altas descargas del Río Chira a la bahía de Paita alteraron significativamente la sedimentación, provocando grandes cambios en la composición de las comunidades bentónicas.

La distribución de las especies de poliquetos por gremios tróficos revela un patrón en relación con el sedimento. Así los depositívoros superficiales (SDF) se encontraron principalmente en fondos arenosos y los suspensívoros (SF) en fondos rocosos someros. Estos resultados contrastan con lo descrito por Sánchez et al. (1993) en el noroeste de la Península Ibérica, quienes determinaron que los SDF fueron dominantes en sedimentos finos con altos contenidos de pellets, debido a su poder de adsorción de la matería orgánica, mientras que los SF prefirieron medios de arenas finas y medias, cuyo hidrodinamismo favorece la entrada de materiales en suspensión.

En nuestro estudio, los poliquetos consumidores de interfase (IF), capaces de cambiar de un comportamiento suspensívoro a uno depositívoro superficial (Gutiérrez, 2000), prefirieron profundidades intermedias de sustrato areno-arcilloso, en tanto que los depositívoros sub-superficiales (SSDF) prefirieron fondos limo-arenosos profundos. Tena et al. (1993) determinaron que el gremio SSDF predomina en zonas donde la energía mecánica que ejercen las corrientes es baja y hay mayor sedimentación, como son los sustratos blandos finos. Por otra parte, aunque algunos autores han señalado cierta correlación positiva entre la presencia de carnívoros y los sedimentos gruesos (Tena et al., 1993), en nuestro caso los omnívoros y depredadores (OP) se presentaron casi uniformemente en todas las texturas.

\section{Agradecimientos}

A la Blga. Soledad Guzmán y al Ing. Octavio Morón por la facilidad y aporte de datos fisico-químicos respectivamente para la elaboración del presente estudio. Este estudio fue financiado y conducido en el marco del proyecto UE-CENSOR (variabilidad del clima y Oscilación Sur del EL Niño: Impactos para los recursos y manejo natural, contrato 511071) y es la publicación CENSOR 0102

\section{Literatura citada}

Ayon Py K. Aronés. 1997. Comunidades de zooplancton e ictioplancton frente a la costa norte-centro del Perú. Crucero BIC Humboldt 9705-06. Inf. Inst. Mar Perú. 128:70-79.

Brey, T., H. Rumohr and S. Ankar. 1988. Energy content of macrobenthic invertebrates: general conversion factors from weight to energy. J. Exp. Mar. Biol. Ecol. 117: 271-278.

Carrit, D. \& J. Carpenter. 1966. Comparision and evaluation of currently employed modifications of the Winkler method for determining disolved oxygen in sea water. J. Mar. Res., 24:286-318.

Dean, W. 1974. Determination of carbonate and organic matter in calcareous sediments by on ignition: comparision with other methods. Jour. Sed. Petrology, 44:242-248.

Delgado, C. y R. Gomero. 1988. Textura, carbono orgánico y carbonatos de los sedimentos del margen continental peruano. En Salzwedel y Landa (eds). Recursos y Dinámica del Ecosistema de Afloramiento Peruano. Bol. Inst. Mar Perú, Vol. Extraordinario: 1-10.

Fauchald, K. y Jumars, P. 1979. The diet of Works: a study of polychaete feeding guilds. Oceanogr. Mar. Bio. I Annu. Rev., 17:191-284

Flores, M., S. Vera, R. Marcelo y E. Chirinos. 1994. Estadisticas de desembarques de la pesqueria marina peruana. 1983-1992. Inf. Inst. Mar. Perú, 105: 1-202. 
Flores, M., S. Vera, R. Marcelo y E. Chirinos. 1997. Estadisticas de desembarques de la pesqueria marina peruana. 1995-1996. Inf. Inst. Mar. Perú, 129: 1-62.

Flores, M., S. Vera, R. Marcelo y E. Chirinos. 1998. Estadisticas de desembarques de la pesqueria marina peruana.1996-1997. Inf. Inst. Mar. Perú, 140: 1-63.

Galehouse, J. 1971. Sedimentation analysis. En Procedures in sedimentary petrology, R.E. Carver (Ed). Wiley, New York, pp:69-94.

Gaston, G. 1987. Benthic polychaeta of the Middle atlantic Bight: feeding and distribution. Marine Ecology Progress Series, 36:251-262.

Gutiérrez, D. 2000. Bioperturbación y Macrofauna en fondos sublitorales de un área de surgencias frente a Chile central $\left(36^{\circ}\right.$ $\left.30^{\prime} \mathrm{S}\right)$ : variación espacial y temporal en el periodo $1997-$ 1999. Tesis para optar al grado de Doctor en Oceanografía. Escuela de Graduados, Universidad de Concepción, Chile. 208pp.

Gutiérrez, D., K. Aronés, F. Chang, L. Quipúzcoa y P. Villanueva. 2005. Impacto de la variabilidad oceanográfica estacional e Inter-anual sobre los ensamblajes de microfitoplancton, mesozooplancton, ictioplancton y macrozoobentos de dos áreas costeras del norte del Perú entre 1994 y 2002. Bol. Inst. Mar Perú, 22: 1-57.

Hidronav-34, 1995. Derrotero de la costa del Perú. Dirección de Hidrografía y Navegación de la Marina. Suplemento $N^{\circ}$ 3. Vol. I.Pp: 58-63.

Ingram, R. 1971. Sieve análisis. En procedures in sedimentology petrology, R.E. Carver (Ed). Wiley, New York, pp:49-67.

Jacinto, M., O. Morón, C. Martínez, P. Villanueva, M. Guzmán y J. Córdova. 1997. Evaluación de la calidad del ambiente marino en el área de Bayovar-Ensenada de Sechura, 8 a 10 Mayo 1996. Inf. Prog. Inst. Mar Perú, 54: 30-64.

Jaimes, E. 1999. condiciones meterológicas a nivel global y local, cambio climático y "El Niño 1997-1998”. En J. Tarazona y E. Castillo (eds.). El Niño 1997-1998 y su impacto sobre los Ecosistemas Marino y Terrestre. Rev. peru. biol., Vol. Extraordinario: 1-8.

Maurer, D., G. Robertson y T. Gerlinger. 1994. Comparison of community structure of soft-bottom macrobenthos of the Newport Submarine Canyon, California and the adjoining Shelf. Int. revue. Ges. Hydrobiol., 79: 591-603.

Mayor, S. 1996. Las comunidades bentónicas entre los $03^{\circ}$ y $09^{\circ} \mathrm{S}$ en mayo de 1995. (Cr. BIC SNP-1, 9505-06). Inf. Inst. Mar Perú, 117: 61-71.

Mayor, S., F. Velazco y R Marquina. 1997. Macrozoobentos del mar peruano en el sublitoral de fondo blando durante el verano de 1996. Inf. Inst. Mar. Perú, 126: 21-27.

Levin, L., C. Hugget y K. Wishner. 1991. Control of deep-sea benthic community structure by oxygen and organic-matter gradients in the eastern Pacific Ocean. J. Mar. Res., 49:763-800.

Prentice, I. 1977. Non-metric ordination methods in ecology. J.Ecol. 65:85-94.

Rubio J., M. Rabí y C. Yamashiro. 1996. Evaluación poblacional del recurso concha de abanico (Argopecten purpuratus) en la isla Lobos de Tierra y bahía de Sechura (14 al 23 de octubre de 1995). Inf. Prog. Inst. Mar Perú, 24: 1-18.
Rubio J. y A. Taipe, 1996. Evaluación poblacional de concha de abanico (Argopecten purpuratus) en la isla Lobos de Tierra y bahía de Sechura. 30 de agosto a 10 de setiembre de 1996. Inf. Prog. Inst. Mar Perú, 46: 1-17.

Rubio J., A. Taipe y S. Aguilar. 1997. Evaluación de la población del recurso concha de abanico (Argopecten purpuratus) en la isla Lobos de Tierra y bahía de Sechura. 05-16 junio 1997. Inf. Prog. Inst. Mar Perú, 69: 1-13.

Salzwedel H., L. Flores, E. De Flores, A. Zafra y G. Carbajal. 1988. Macrozoobentos del sublitoral Peruano, Antes Durante, y Después de el Niño 1982-1983. En Salzwedel y Landa (eds). Recursos y Dinámica del Ecosistema de Afloramiento Peruano. Bol. Inst. Mar Perú, Vol. Extraordinario: 77-98.

Sánchez, A., J. Mora, J. Garmendia y M. Lastra. 1993. Estructura trófica del macrozoobentos submareal de la ría de AresBetanzos. I: Composición y distribución. Publ. Espec. Inst. Esp. Oceanogr., 11: 33-40.

Siegel, S. 1994. Estadística no paramétrica: aplicada a las ciencias de la conducta. 3ra ed. Ed. Triller. Mexico. 344 p.

Sneath, P. \& R. Sokal. 1973. Numerical taxonomy. W. H. Freeman, San francisco. 571pp.

Tafur, R., G. Castillo, A. Taipe, L. Vásquez, E. Delgado y N. Carrasco. 2000. Evaluación poblacional de la concha de abanico (Argopecten purpuratus) en Bahía de Sechura e Isla Lobos de Tierra. Julio 1999. Inf. Prog. Inst. Mar Perú, 113: 1-39.

Tarazona, J. 1990. Disturbance and stress associated to El Niño and their significance for the macrobentos of shallow areas of the peruvian upwelling ecosystem. Ph.D. thesis. University of Bremen. 181 pp.

Tarazona, J., W. Arntz, E. Canahuire, Z. Ayala y A. Robles. 1988. Modificaciones producidas durante "El Niño" en la infauna bentónica de Áreas someras del Ecosistema de Afloramiento Peruano. Conferencia del Symposium "El Fenómeno El Niño y su impacto en la fauna marina", dentro del Noveno Congreso Latinoamericano de Zoología. Boletín Inst. Mar Perú. Vol. Extraordinario: 55-64.

Tarazona, J., W. Arntz and E. Canahuire. 1996. Impact of Two "El Niño" Events of Different Intensity of the Hypoxic Soft Bottom Macrobenthos off the Central Peruvian Coast. Marine Ecology, 17(1-3): 425-446.

Tarazona, J., A. Indacochea, S. Valle, C. Córdova, N. Ochoa, W. Serrano y T. Peña. 2000. Impacto de "El Niño 1997-1998" sobre el ecosistema marino somero de la costa central del Perú. En J. Tarazona y E. Castillo (eds.). El Niño 1997-1998 y su impacto sobre los Ecosistemas Marino y Terrestre. Rev. peru. biol., Vol. Extraordinario: 18-31.

Tena, J., R. Capaccioni-Azzati, F. Torres-Gavila y R. Porras. 1993. Anélidos poliquetos del antepuerto de Valencia: distribución y categorías tróficas. Publ. Espec. Inst. Esp. Oceanogr., 11: $15-20$

Valentin, J. 2000. Ecología Numérica: Uma introducao a analise multivariada de dados ecológicos. Editora Interciencia. Río de Janeiro 2000. 115 pp.

Wheatcroft, R., I Olmez and F. Pink. 1994. Particle bioperubation in Massachusetts Bay: Preliminary results using and new deliberate tracer technique. J. Mar. Res. 52: 1129-1150. 
